\title{
Computation of the Best Approximations for $\theta$ - Expansion of Continued Fractions using Maple
}

\author{
Khairun Nisak Muhammad ${ }^{1}$ and Hailiza Kamarulhaili ${ }^{2}$ \\ ${ }^{1}$ Department of Mathematics, Faculty of Science, Universiti Putra Malaysia, 43400 UPM Serdang, Selangor, \\ Malaysia \\ ${ }^{1,2}$ School of Mathematical Sciences, Universiti Sains Malaysia, 11800 USM Penang, Malaysia
}

\begin{abstract}
We considered an expansion of real numbers $x=\left[a_{1} \theta, a_{2} \theta, \ldots, a_{n} \theta+1 / R_{n}\right]$ with $R_{n}=\left[a_{n+1} \theta, a_{n+2} \theta, \ldots\right]$ where $x \in(0, \theta)$ and $0<\theta<1$. This paper emphasizes the best approximations of $\theta$-expansions since we knew the best approximations of regular continued fraction (RCF) expansions has been generally proved as $C_{n}$. Regarding this purpose, we provided the numerical computations on the $\theta$-convergent based on a few samples by using a Maple software. This software helped us to compute the value of $\theta$ convergent briskly and efficiently instead of using the traditional method of computing the convergent of a small sample involving the Euclidean algorithm. We took various values of $\theta$ within the stated range including $\theta=1$. This is because, we were eager to know how the value of $\theta$ influenced the value of convergent for both $\theta$ and RCF expansions. Hence, throughout the numerical experiment conducted, we revealed that in general, there was no specific $\theta$-convergent to be the best approximations of $x$ in each sample. The samples might give different best approximations and the results were depending on the values of their $\theta$. However, similar things that happened in every sample of $\theta$-expansions are their best approximations always occurred early in the sequence.
\end{abstract}

Keywords: $\theta$-convergent, $\theta$-expansions, best approximations, RCF-convergent, RCF expansions

\section{PRELIMINARIES}

Suppose that throughout our discussion, we referred our expansion as the continued fraction expansion of a number $x$ in $(0, \theta)$. One expansion discussed by (Chakraborty \& Rao, 2003), which was studied in detail by (Sebe \& Lascu, 2014), had raised a different type of continued fractions, namely $\theta$-expansions. (Chakraborty \& Rao, 2003) defined this new expansion as follows:

Definition 1 Let $x>0$. Let $a_{0}=\max \{n \geq 0 ; n \theta \leq x\}$. If $x$ already equals $a_{0} \theta$, we write $x=\left[a_{0} \theta\right]$. Otherwise, define $r_{1}$ by $x=a_{0} \theta+1 / r_{1}$ where $0<1 / r_{1}<\theta$. Then, $r_{1}>$ $1 / \theta \geq \theta$ and let $a_{1}=\max \left\{n \geq 0 ; n \theta \leq r_{1}\right\}$. If $r_{1}=a_{1} \theta$, then we write $x=\left[a_{0} \theta, a_{1} \theta\right]$, in other word, $x=a_{0} \theta+1 / a_{1} \theta$. If $a_{1} \theta<r_{1}$, define $r_{2}$ by $r_{1}=a_{1} \theta+1 / r_{2}$ where $0<1 / r_{2}<\theta$. So, $r_{2}>1 / \theta \geq \theta$ and let $a_{2}=\max \left\{n \geq 0 ; n \theta \leq r_{2}\right\}$. To proceed this way, either the process terminates at, say, $n$ steps or it continues indefinitely. In the former case, we write $x=\left[a_{0} \theta ; a_{1} \theta, \ldots, a_{n} \theta\right]$ and we call this the continued fraction expansion of $x$ with respect to $\theta$ terminating at the $n$-th stage. In the latter case, we write $x=$ $\left[a_{0} \theta ; a_{1} \theta, a_{2} \theta, \ldots, a_{n} \theta+1 / R_{n}\right]$ where $R_{n}=\left[a_{n+1} \theta, a_{n+2} \theta, \ldots\right]$ and it is called the infinite or non-terminating continued fraction expansion of $x$ with respect to $\theta$.

When $x<\theta$, we will have $a_{0}=0$ and instead of writing $x=\left[0 ; a_{1} \theta, a_{2} \theta, \ldots, a_{n} \theta+1 / R_{n}\right]$, we can also write $x=$ 
$\left[a_{1} \theta, a_{2} \theta, \ldots, a_{n} \theta+1 / R_{n}\right]$ which is the same as following expansion:

$$
x=\frac{1}{a_{1} \theta+\frac{1}{a_{2} \theta+} . \underset{+\frac{1}{a_{n} \theta+\frac{1}{R_{n}}}}{ }} .
$$

where

$$
R_{n}=a_{n+1} \theta+\frac{1}{a_{n+2} \theta+\cdots} .
$$

From expansion in (1), Chakraborty \& Rao (2003) also defined the following item:

\section{Definition 2 Define the $n$-th $\theta$-convergent of a}

number $x \in(0, \theta)$ as

$$
C_{n}=\frac{p_{n}}{q_{n}}=\left[a_{1} \theta, a_{2} \theta, \ldots, a_{n} \theta\right]
$$

for $n \geq 0$.

From Definition 2, in case $x$ has terminating expansion, say, $x=\left[a_{1} \theta, a_{2} \theta, \ldots, a_{k} \theta\right]$, then clearly $p_{k} / q_{k}=x$. We make the usual convention that in this case,

$$
\frac{p_{n}}{q_{n}}=x
$$

for $n \geq k$.

Next, in this project, we let our $0<x<\theta$ with $x=$ $\left[a_{1} \theta, a_{2} \theta, \ldots, a_{n} \theta+1 / R_{n}\right]$. In what follows $a_{n}, p_{n}$ and $q_{n}$ depends on $x$. The stated identities hold for all $n$ in case $x$ has non-terminating expansion and they hold for $n \leq k$ in case $x$ has expansion terminating at the $k$-th stage. Then, following are the recurrence relations for $n \geq 1$, as follows:

$$
\begin{aligned}
& \quad p_{n}=a_{n} \theta p_{n-1}+p_{n-2} \\
& q_{n}=a_{n} \theta q_{n-1}+q_{n-2} .
\end{aligned}
$$

Following the convention of the usual continued fraction expansions, namely, $p_{-1}=1, p_{0}=0, q_{-1}=0, q_{0}=1$, we obtain for the $\theta$-expansions,

$$
\begin{aligned}
& p_{0}=a_{0} \theta=0 ; p_{1}=a_{0} \theta a_{1} \theta+1 \\
& q_{0}=1 ; q_{1}=a_{1} \theta .
\end{aligned}
$$

From (1), (2), (5) and (6), we obtain that

$$
x=\frac{p_{n}+\frac{1}{R_{n}} p_{n-1}}{q_{n}+\frac{1}{R_{n}} q_{n-1}} .
$$

(Chakraborty \& Rao, 2003) have initiated this $\theta$ expansions motivated by problems in random continued fraction expansions including the RCF expansions as well. The motivation of this research focus on $\theta$-expansion is because we would like to critically examine the behaviors of $\theta$-expansions and observe how various values of $\theta$ really affecting the result of their convergent if we compared to the result and convergent of RCF expansions.

In this paper, our concern was to observe and discuss the best approximations of $\theta$-expansions since we knew for RCF expansions, $C_{n}$ will always be the best approximations. Recently, afew papers have discussed on the properties of the $\theta$-convergent as in the paper written by Muhammad \& Kamarulhaili, (2018). They demonstrated a detailed study on the convergent properties for a family of $\theta$-expansions. In addition, they also observe the pattern and behaviors of those convergent besides provide the numerical computations on the $\theta$ convergent.

Actually, RCF expansions act as a pillar to the development of $\theta$-expansions. Hence, Muhammad \& Kamarulhaili, (2016) have provided the properties of RCFconvergent which then helped to generate the behaviours of $\theta$-expansions. In that paper, they discussed the properties that are relevant to sequences of integer in RCF expansions which involved the concept of Euclidean algorithm, extended Euclidean algorithm, and continued fraction algorithm. Those properties are regarded as part and parcel of the building blocks of a new generation of an efficient cryptographic protocol. Also, continued fraction algorithm is widely used in cryptography such as in (Thirumalai et al., 2016).

From the convergent, we were likely to perceive how fast does a continued fraction converge and how fast it obtains the best approximations. With regard to this matter, for RCF-expansions have been answered and provided through the research made by Falbo (2018). On the other hand, there were a few theorems of a general character for the convergence of continued fraction with complex elements as discussed and obtained by Van Vleck, (1901).

Besides, continued fraction is also applied in graph theory. Bouttier \& Guitter (2012) presented an unexpected connection between two map enumeration problems. They showed that in the general class of maps with controlled face degrees, the solution for both problems are actually encoded into the same quantity, respectively via its power 
series expansion and its continued fraction expansion.

This paper has been organized into 4 sections. In the first section, we introduced our preliminaries on the convergent for both $\theta$ and RCF expansions. Next, in Section 2, we provided the numerical results for both expansions. We discussed the mathematical programming, samples used, computation and the comparisons of the best approximations on both expansions. Then, we provided the behaviours of $\theta$ expansions in Section 3 and last but not least, in Section 4, we summarized the overall research in this paper.

\section{NUMERICAL RESULTS AND DISSCUSSIONS}

In this section, we discuss on the results of numerical computations for both $\theta$-expansions and RCF-expansions based on our samples by using the mathematical programming. We then examine the pattern based on the convergent computed. Next, we observe and provide their best approximations and compare the best approximations between these both expansions.

\section{A. Computation of Irrational Numbers and Mathematical Programming}

This project focus on the infinite expansions which involving computation of the irrational numbers. Thus, Strayer (2001) provide us one proposition of constructing the infinite continued fraction.

Proposition 1 Let $x \in \mathbb{R}$. Then, $x \in \mathbb{R}-\mathbb{Q}$ if and only if $x$ is expressible as an infinite regular continued fraction.

Hence, through this proposition, we let $x=x_{0} \in \mathbb{R}-\mathbb{Q}$. We defined $a_{0}, a_{1}, a_{2}, \ldots$ and $x_{1}, x_{2}, \ldots$ by the following recurrence relations:

$$
\begin{array}{r}
a_{i}=\left[x_{i}\right], i \geq 0 \\
x_{i+1}=\frac{1}{x_{i}-a_{i}}, i \geq 0 .
\end{array}
$$

Then, we used the Maple software to compute our samples based on Proposition 1 since this project involved the computation of large samples of irrational value, $x$ for both $\theta$ and RCF expansions. First, we obtain its $a_{n}$ 's which then, we substituted the value of various thetas in the range of $0<\theta \leq 1$ and lastly we obtain the value of their $\theta$-convergent for each sample.

Throughout the project, we have analysed more than 50 samples of $x$ and used various values of $\theta$. The purpose was to observe the performance of different values of $\theta$ on a fixed value of $x$ towards the value of convergent. However, in this paper, we provided 2 samples of approximated value of $x$ with iterations, $n=10$ and used 10 different values of $\theta$.

\section{B. Convergent for Both Expansions}

We provided two samples for computing the $\theta$ and RCF convergent with iteration, $n=10$. For these two samples, we used ten different values of $\theta$.They were $\theta_{1}=0.1, \theta_{2}=$ $0.2, \theta_{3}=0.3, \theta_{4}=0.4, \theta_{5}=\{\sqrt{21}\}=0.582575695, \theta_{6}=$ $\{\sqrt{13}\}=0.605551275, \quad \theta_{7}=0.7, \quad \theta_{8}=0.8, \quad \theta_{9}=\{\sqrt{35}\}=$ 0.916079783 , and $\theta_{10}=1.00$. For the first sample, we let our approximated value, $x=\{\sqrt{18}\}=0.242640686$.By applying equation (3), (5), (6) and (8) on Maple, we obtained their $a_{n} \theta_{n}, p_{n}, q_{n}$ and $C_{n}$. The convergent of both expansions are stated below:

Table 1 . The value of convergent, $C_{n}$ for ten different values of theta, $\theta_{n}$ for $x=0.242640686$.

\begin{tabular}{|c|c|c|c|c|c|}
\hline \multirow{2}{*}{$C_{n}$} & \multicolumn{5}{|c|}{ Theta, $\theta_{n}$} \\
\cline { 2 - 6 } & $\theta_{1}=0.1$ & $\theta_{2}=0.2$ & $\theta_{3}=0.3$ & $\theta_{4}=0.4$ & $\theta_{5}=\{\sqrt{21}\}$ \\
\hline$C_{1}$ & 2.500000000 & 1.250000000 & 0.833333333 & 0.625000000 & 0.429128785 \\
\hline$C_{2}$ & 0.706060606 & 0.701754386 & 0.618556701 & 0.522875817 & 0.392947817 \\
\hline$C_{3}$ & 1.422413793 & 0.868902439 & 0.662568306 & 0.537219101 & 0.395761131 \\
\hline$C_{4}$ & 0.899922421 & 0.810076562 & 0.653046659 & 0.535157278 & 0.395540933 \\
\hline$C_{5}$ & 1.176524279 & 0.829849156 & 0.655083339 & 0.535452687 & 0.395558159 \\
\hline$C_{6}$ & 0.993341417 & 0.821765663 & 0.654539897 & 0.535398802 & 0.395556408 \\
\hline$C_{7}$ & 1.148890591 & 0.827515070 & 0.654852845 & 0.535423586 & 0.395556944 \\
\hline$C_{8}$ & 1.019144000 & 0.823453574 & 0.654661625 & 0.535410464 & 0.395556726 \\
\hline$C_{9}$ & 1.129019301 & 0.826336629 & 0.654774497 & 0.535417074 & 0.395556807 \\
\hline$C_{10}$ & 1.000717467 & 0.823802877 & 0.654681953 & 0.535412055 & 0.395556754 \\
\hline \multirow{2}{*}{$C_{n}$} & & & Theta, $\theta_{n}$ & & \\
\cline { 2 - 7 } & $\theta_{6}=\{\sqrt{13}\}$ & $\theta_{7}=0.7$ & $\theta_{8}=0.8$ & $\theta_{9}=\{\sqrt{35}\}$ & $\theta_{10}=1.00$ \\
\hline$C_{1}$ & 0.412846955 & 0.357142857 & 0.312500000 & 0.272901995 & 0.250000000 \\
\hline$C_{2}$ & 0.380426511 & 0.335731415 & 0.297951583 & 0.263104573 & 0.242424242 \\
\hline$C_{3}$ & 0.382787082 & 0.336942469 & 0.298598754 & 0.263444119 & 0.242647059 \\
\hline$C_{4}$ & 0.382614218 & 0.336873738 & 0.298569906 & 0.263432337 & 0.242640500 \\
\hline$C_{5}$ & 0.382626871 & 0.336877638 & 0.298571192 & 0.263432745 & 0.242640693 \\
\hline$C_{6}$ & 0.382625665 & 0.336877348 & 0.298571117 & 0.263432727 & 0.242640685 \\
\hline$C_{7}$ & 0.382626017 & 0.336877417 & 0.298571134 & 0.263432730 & 0.242640686 \\
\hline$C_{8}$ & 0.382625879 & 0.336877394 & 0.298571127 & 0.263432729 & 0.242640686 \\
\hline$C_{9}$ & 0.382625928 & 0.336877401 & 0.298571128 & 0.263432729 & 0.242640686 \\
\hline$C_{10}$ & 0.382625897 & 0.336877397 & 0.298571127 & 0.263432729 & 0.242640686 \\
\hline & & & & \\
\end{tabular}

For the second sample, we let our approximated value, $x=\{\sqrt{91}\}=0.539392014$ with ten different values of $\theta$ as 
stated earlier. Again, by applying the method in(3), (5), (6) and (8) on Maple, we obtained their $a_{n} \theta_{n}, p_{n}, q_{n}$ and $C_{n}$. The convergent of both expansions are shown in Table 2.

Table 2. The value of convergent, $C_{n}$ for ten different values of theta, $\theta_{n}$ for $x=0.539392014$.

\begin{tabular}{|c|c|c|c|c|c|}
\hline \multirow{2}{*}{$C_{n}$} & \multicolumn{5}{|c|}{ Theta, $\theta_{n}$} \\
\cline { 2 - 6 } & $\theta_{1}=0.1$ & $\theta_{2}=0.2$ & $\theta_{3}=0.3$ & $\theta_{4}=0.4$ & $\theta_{5}=\{\sqrt{21}\}$ \\
\hline$C_{1}$ & 10.00000000 & 5.000000000 & 3.333333333 & 2.500000000 & 1.716515139 \\
\hline$C_{2}$ & 0.099009901 & 0.192307692 & 0.275229358 & 0.344827586 & 0.434954542 \\
\hline$C_{3}$ & 1.735537190 & 0.967741936 & 0.749354005 & 0.661764706 & 0.601456455 \\
\hline$C_{4}$ & 0.391499299 & 0.491614907 & 0.554399880 & 0.549822064 & 0.545022133 \\
\hline$C_{5}$ & 1.010743258 & 0.648734177 & 0.574790294 & 0.560866962 & 0.560843135 \\
\hline$C_{6}$ & 0.270361537 & 0.428189117 & 0.499796019 & 0.558876285 & 0.556131743 \\
\hline$C_{7}$ & 0.941068283 & 0.621328012 & 0.562143041 & 0.559388705 & 0.559307979 \\
\hline$C_{8}$ & 0.710800088 & 0.592900322 & 0.556264627 & 0.559238618 & 0.559147147 \\
\hline$C_{9}$ & 0.891529199 & 0.608314420 & 0.558372611 & 0.559275210 & 0.559168779 \\
\hline$C_{10}$ & 0.718827624 & 0.594138952 & 0.556524017 & 0.559245008 & 0.559153241 \\
\hline \multirow{2}{*}{$C_{n}$} & & & $T h 40, \theta_{n}$ & & \\
\cline { 2 - 7 } & $\theta_{6}=\{\sqrt{13}\}$ & $\theta_{7}=0.7$ & $\theta_{8}=0.8$ & $\theta_{9}=\{\sqrt{35}\}$ & $\theta_{10}=1.00$ \\
\hline$C_{1}$ & 1.651387820 & 1.428571429 & 1.250000000 & 1.091607978 & 1.000000000 \\
\hline$C_{2}$ & 0.443077973 & 0.469798658 & 0.487804878 & 0.498085419 & 0.500000000 \\
\hline$C_{3}$ & 0.597327766 & 0.583262890 & 0.570652174 & 0.556296671 & 0.545454546 \\
\hline$C_{4}$ & 0.547598133 & 0.543236835 & 0.542603613 & 0.545997982 & 0.538461539 \\
\hline$C_{5}$ & 0.561017523 & 0.560168607 & 0.556148591 & 0.547686864 & 0.539473684 \\
\hline$C_{6}$ & 0.557195495 & 0.556512416 & 0.555437055 & 0.547406276 & 0.539325843 \\
\hline$C_{7}$ & 0.559722083 & 0.558519937 & 0.555832195 & 0.547546122 & 0.539393939 \\
\hline$C_{8}$ & 0.559598677 & 0.558477098 & 0.555817510 & 0.547541627 & 0.539391958 \\
\hline$C_{9}$ & 0.559614221 & 0.558481293 & 0.555818643 & 0.547541898 & 0.539392060 \\
\hline$C_{10}$ & 0.559603271 & 0.558478575 & 0.555817974 & 0.547541755 & 0.539392010 \\
\hline
\end{tabular}

Next, we provide the graph for growth rate of convergent for these two samples. The graphs are plotted as follow:

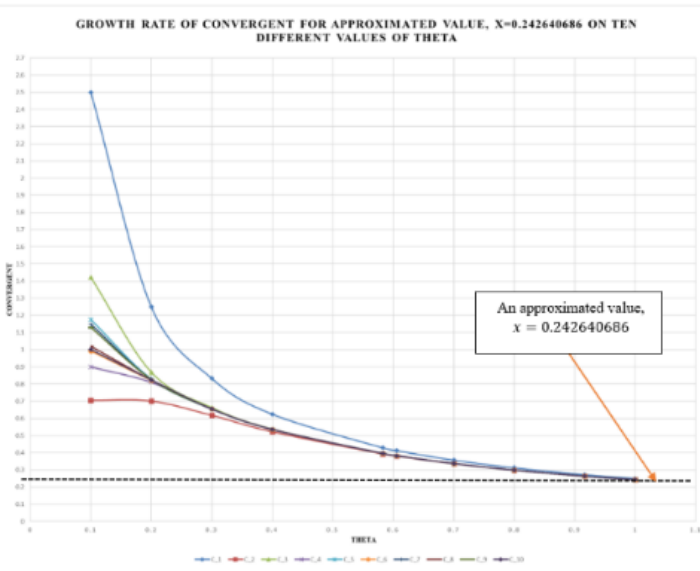

Figure 1: Growth rate of convergent for approximated value, $x=0.242640686$.

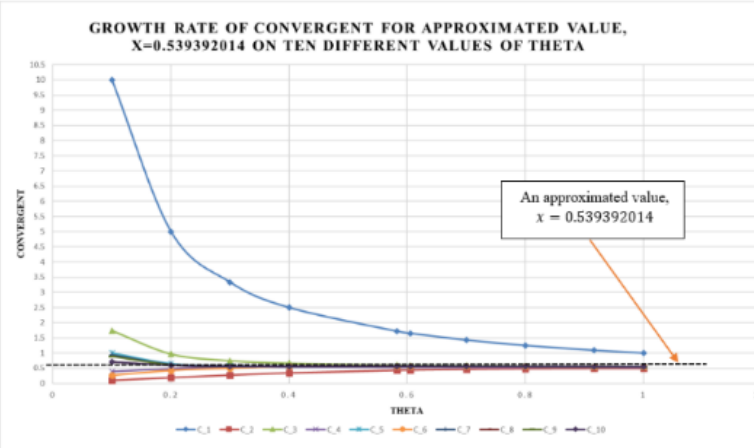

Figure 2: Growth rate of convergent for approximated value, $x=0.539392014$

\section{Best Approximations on Both Expansions}

Based on our samples, we are going to discuss their best approximations of $\theta$-expansions and RCF expansions. We aimed to investigate and observe the impact of different values of $\theta$ on the values of convergent. Table I presents the convergent of the approximated value, $x=\{\sqrt{18}\}=$ 0.242640686. For $\theta$-expansions with $0<\theta<1$, we obtained that for each of $x, C_{2}$ was the closest $\theta$-convergent values to the best decimal approximation of $x$. In addition, through the graph in Figure 1, we can see that as $n \rightarrow \infty$, $C_{2} \rightarrow x$. However, for RCF expansions when $\theta=1.00$; starting from $C_{7}$, the value of RCF-convergent started to converge the closest and exactly the same as the approximated value of $x$.

Next, Table II illustrates that for approximated value $x=\{\sqrt{91}\}=0.539392014, C_{4}$ was the closest $\theta$-convergent. However, it was different for RCF expansions when $\theta=$ 1.00 , as $n \rightarrow \infty, C_{n} \rightarrow x$. Figure 2 shows the value of RCFconvergent was almost similar to the best decimal approximation of $x$.

As a conclusion, From Table I, II, Figure 1 and 2, we can conclude that as the value of $\theta$ approaches to 1 , the closer the value of $\theta$-convergent to the approximated value of $x$. We provide this theoretical prove in the next section. Apart from that, we found that there was no specific $\theta$ convergent to be the best approximations of $x$ for $\theta$ expansions in general for each sample. Every sample might give different best approximations and the results are depending on the value of its theta, $\theta$. However, 
similar things that happen in every sample are the best approximations always occur early in the sequence.

\section{Comparisons on the Best Approximations between Both Expansions}

Following were similarities and differences effects on the convergent for both expansions:

Table 3. The similarities between $\theta$-expansions and RCF

\begin{tabular}{|c|c|c|}
\hline Expansion & $\boldsymbol{\theta}$ - & RCF \\
& Expansions & Expansions \\
\hline Similarities & For $n \geq 1$, as $\theta \rightarrow 1$, each $C_{n} \rightarrow x$ \\
\hline
\end{tabular}

Table 4. The differences between $\theta$-expansions and RCF

\begin{tabular}{|c|c|c|}
\hline $\begin{array}{c}\text { Expansio } \\
\text { ns }\end{array}$ & $\theta$-Expansions & $\begin{array}{c}\text { RCF } \\
\text { Expansions }\end{array}$ \\
\hline $\begin{array}{c}\text { Converge } \\
\text { nt } \\
\text { converge }\end{array}$ & $\begin{array}{l}\theta \text {-convergent will } \\
\text { converge close to } \\
\text { the } \\
\text { approximated } \\
\text { value of } x \text { at the } \\
\text { early iteration of } \\
\text { convergent }\end{array}$ & $\begin{array}{l}\text { As } n \rightarrow \infty \text {, we will } \\
\text { obtain the } \\
\text { closest RCF- } \\
\text { convergent and } \\
\text { the value of their } \\
\text { convergent is } \\
\text { exactly the same } \\
\text { with the } \\
\text { approximated } \\
\text { value of } x\end{array}$ \\
\hline $\begin{array}{c}\text { Range of } \\
\text { Theta, } \theta\end{array}$ & $0<\theta<1$ & $\theta=1.00$ \\
\hline $\begin{array}{c}\text { Best } \\
\text { approxim } \\
\text { ations }\end{array}$ & $\begin{array}{c}\text { No exact best } \\
\text { approximations } \\
\text { for } \theta-C_{n} \text {. It is all } \\
\text { depending on the } \\
\text { value of } \theta\end{array}$ & $\begin{array}{c}C_{n} \text { is always the } \\
\text { best } \\
\text { approximations } \\
\text { for RCF } \\
\text { expansions }\end{array}$ \\
\hline
\end{tabular}

Based on the previous numerical computations, we provide these following theoretical behaviours to support the statement.

\section{THEORETICAL BEHAVIORS OF $\theta$ -} EXPANSIONS

Lemma 1 Let $x=\left[a_{1} \theta, a_{2} \theta, \ldots, a_{n} \theta+1 / R_{n}\right]$ where $_{n}=$ $\left[a_{n+1} \theta, a_{n+2} \theta, \ldots\right]$ be an infinite $\theta$-expansion of continued fraction and let all notation be as in equation (5)and(6).
Then for $n \geq 0$,

$$
p_{n-1} q_{n}-p_{n} q_{n-1}=(-1)^{n} .(9)
$$

\section{Proof:}

We used the method of induction on $n$ to prove this property. If $n=1$, we have

$$
p_{0} q_{1}-p_{1} q_{0}=a_{0} \theta a_{1} \theta-a_{0} \theta a_{1} \theta-1=-1 .
$$

Now, we assumed that $m \geq 1$ and the desired result holds for $n=m$, so that

$$
p_{m-1} q_{m}-p_{m} q_{m-1}=(-1)^{m} .
$$

We must show that

$$
p_{m} q_{m+1}-p_{m+1} q_{m}=(-1)^{m+1}
$$

so that the desired result holds for $n=m+1$. We have

$$
p_{m} q_{m+1}-p_{m+1} q_{m}
$$

$$
\begin{aligned}
& =p_{m}\left(a_{m+1} \theta q_{m}+q_{m-1}\right) \\
& -\left(a_{m+1} \theta p_{m}+p_{m-1}\right) q_{m} \\
& =-\left(p_{m-1} q_{m}-p_{m} q_{m-1}\right)=-(-1)^{m} \\
& =(-1)^{m+1}
\end{aligned}
$$

asdesired

Theorem 1 Let $x=\left[a_{1} \theta, a_{2} \theta, \ldots, a_{n} \theta+1 / R_{n}\right]$ where $R_{n}=\left[a_{n+1} \theta, a_{n+2} \theta, \ldots\right]$ be an infinite $\theta$-expansion of continued fraction with $x \in(0, \theta)$ and fixed $\theta$ within the range of $0<\theta<1$. The $\theta$-convergent, $p_{n}$ and $q_{n}$ are as stated in recurrence relations (3), (5) and (6). If $p_{n} / q_{n}$ satisfying

$$
\left|x-\frac{p_{n}}{q_{n}}\right|<\left|x-\frac{p_{n-1}}{q_{n-1}}\right|
$$

then $q_{n}$ form an increasing sequence withrestriction $\left(R_{n-1} q_{n-1}+q_{n-2}\right)>\left(R_{n} q_{n}+q_{n-1}\right)$.

Proof:

From (7) and (9), we have $x=$ $\left(p_{n} R_{n}+p_{n-1}\right) /\left(q_{n} R_{n}+q_{n-1}\right) \quad$ and $\quad p_{n-1} q_{n}-p_{n} q_{n-1}=$ $(-1)^{n}$. Then, we obtain

$$
\left|x-\frac{p_{n}}{q_{n}}\right|=\left|\frac{(-1)^{n}}{R_{n} q_{n}^{2}+q_{n-1} q_{n}}\right|
$$

where $R_{n}=\left[a_{n+1} \theta ; a_{n+2} \theta, \ldots\right]$. Then,

$$
\left|x-\frac{p_{n-1}}{q_{n-1}}\right|=\left|\frac{(-1)^{n-1}}{R_{n-1} q_{n-1}^{2}+q_{n-1} q_{n-2}}\right| .
$$

Hence, we compared(15)and(16), we obtained as follows:

$$
\begin{aligned}
&\left|\frac{(-1)^{n}}{R_{n} q_{n}^{2}+q_{n-1} q_{n}}\right|<\left|\frac{(-1)^{n-1}}{R_{n-1} q_{n-1}{ }^{2}+q_{n-1} q_{n-2}}\right| \\
& \frac{1}{R_{n} q_{n}^{2}+q_{n-1} q_{n}}<\frac{1}{R_{n-1} q_{n-1}{ }^{2}+q_{n-1} q_{n-2}} \\
& R_{n} q_{n}^{2}+q_{n-1} q_{n}>R_{n-1} q_{n-1}{ }^{2}+q_{n-1} q_{n-2}
\end{aligned}
$$




$$
\begin{gathered}
q_{n}\left(R_{n} q_{n}+q_{n-1}\right)>q_{n-1}\left(R_{n-1} q_{n-1}+q_{n-2}\right) \\
\frac{q_{n}}{q_{n-1}}>\frac{\left(R_{n-1} q_{n-1}+q_{n-2}\right)}{\left(R_{n} q_{n}+q_{n-1}\right)} \\
q_{n}>q_{n-1}
\end{gathered}
$$

with restriction $\left[\left(y_{n} q_{n-1}+q_{n-2}\right) /\left(y_{n+1} q_{n}+q_{n-1}\right)\right]>$

1.

Theorem 2 Let $x=\left[a_{1} \theta, a_{2} \theta, \ldots, a_{n} \theta+1 / R_{n}\right]$ where $R_{n}=\left[a_{n+1} \theta, a_{n+2} \theta, \ldots\right]$ be an infinite $\theta$-expansion of continued fraction with $x \in(0, \theta)$ and fixed $\theta$ within the range of $0<\theta<1$. Every $\theta$-convergent, $p_{n} / q_{n}$ are satisfied as in the recurrence relations(3), (5) and (6). As $\theta \rightarrow 1$, the $\theta$-convergent will tend to the best approximated value of $x$.

\section{Proof:}

We have $p_{n}$ and $q_{n}$ satisfying the recurrence relations (5) and (6). From (15), we have $\left|x-\left(p_{n} / q_{n}\right)\right|=$ $\left|(-1)^{n} /\left(R_{n} q_{n}^{2}+q_{n-1} q_{n}\right)\right|$. We expanded equation (15), then we obtained

$$
\begin{gathered}
\left|x-\frac{p_{n}}{q_{n}}\right| \\
=\left|\frac{(-1)^{n}}{R_{n}\left(a_{n} \theta q_{n-1}+q_{n-2}\right)^{2}+\left(a_{n} \theta q_{n-1}+q_{n-2}\right) q_{n-1}}\right| .
\end{gathered}
$$

From (18), obviously, when the value of $\theta$ increases, it will lead to the increase in value to the denominator of equation (18). Then,increase in the value of $\theta$ will make the right-hand side of equation (18)tends to approach 0 where it automatically gives the smallest distance between its $\theta$-convergent to the best approximated value of $x$, in which, we have $\left|x-C_{n}\right| \rightarrow 0$ as $\theta \rightarrow 1$. Thus, as the value of $\theta$ increases, its $\theta$-convergent will tend to the best approximated value of $x$.

Definition 3Given an infinite $\theta$-expansionx $=$ $\left[a_{1} \theta, a_{2} \theta, \ldots, a_{n} \theta+1 / R_{n}\right]$ where $R_{n}=\left[a_{n+1} \theta, a_{n+2} \theta, \ldots\right]$ of continued fraction with $\theta$-convergent, $C_{n}=p_{n} / q_{n}$. $C_{n}=p_{n} / q_{n}$ is the best approximate decimal number if the distance of $\left|x-p_{n} / q_{n}\right|$ gives the smallest value and is the closest to the value of $x$.

\section{SUMMARY}

Throughout this paper, we have provided the theoretical properties of $\theta$-expansions to support their numerical evidence. Basically, the idea for this expansion was generated from RCF expansions. However, from our observations, the pattern of $\theta$-expansions was not likely the same as RCF expansions since the value of $\theta$ gave a different view on the growth rate of convergent for both expansions. In our project, we applied the Maple software to compute the convergent of big samples of irrational number, $x$.

Hence, from the numerical results, we were likely to perceive the best decimal approximations of both $\theta$ and RCF expansions. The best decimal approximations of $\theta$ expansions was totally different from the best approximations of RCF expansions.For $\theta$-expansions, the best decimal approximations are depending on the value of $\theta$ and there is no specific pattern to determine their best approximations since the value of $\theta$ are varies.From our numerical calculations, we found that for $\theta$-expansions, in most of the cases, $C_{2}$ gave the best approximations within the range of $0<\theta<1$.However, there are certain cases in which $C_{4}$ is the best approximations in the range of $0<$ $\theta<1$. Then, for RCF expansions, $C_{n}$ is always their best approximations.

\section{ACKNOWLEDGEMENT}

The authors would like to express their sincere gratitude to the RU (Research University) Grant, Universiti Sains Malaysia Account No. 1001/PMATHS/811337 for the financial support to carry out this research. 


\section{REFERENCES}

Chakraborty, S. and Rao, B. V. (2003). $\theta$-expansions and the generalized Gauss map. Lecture NotesMonograph Series, 49-64.

Bouttier, J. and Guitter, E. (2012). Planar maps and continued fractions. Communications in Mathematical Physics, 309(3), 623-662.

Falbo, C. E. (2018) How fast does a continued fraction converge?, viewed 5 May 2019 , <https://www.mathfile.net/resources-links>

Muhammad, K. N. and Kamarulhaili, H. (2016, June). On the sequences $r_{i}, s_{i}, t_{i} \in \mathbb{Z}$ related to extended Euclidean algorithm and continued fractions. In AIP Conference Proceedings (Vol. 1739, No. 1, p. 020002). AIP Publishing.
Muhammad, K. N. and Kamarulhaili, H. (2018, June). On the convergent of $\theta$-expansions of continued fractions. In AIP Conference Proceedings (Vol. 1974, No. 1, p. 030002). AIP Publishing Sebe, G. I. and Lascu, D. (2014). A Gauss-Kuzmin theorem and related questions for $\theta$ expansions. Journal of Function Spaces, 2014.

Strayer, J. K. (2001). Elementary number theory. Waveland Press.

Thirumalai, C. S., Senthilkumar, M., Silambarasan, R. and Westphall, C. B. (2016). Analyzing the strength of Pell's RSA. International journal of Pharmacy and Technology, 8(4), 21869-74.

Van Vleck, E. B. (1901). On the convergence of continued fractions with complex elements. Transactions of the American Mathematical Society, 2(3), 215-233. 